\title{
Study on the Agent-based Modeling Method and Simulation System of Consumers Respondence to the Price of Electric Power in Smart Grid
}

\author{
Peng $\operatorname{Han}^{1, a^{*}}$, Qian Feng ${ }^{1, b}$ \\ ${ }^{1}$ School of Computer \& Communication engineering \\ Northeastern University at Qinhuangdao, Hebei, China. 066004 \\ a email: hp@mail.neuq.edu.cn, bemail: mushengge@126.com
}

Keywords: Smart Grid; Consumers; Electric Price Strategy; Agent-based Modeling; Simulation System

\begin{abstract}
This paper involved two parts: the agent-based modeling method and simulation system of the consumers in smart grid, to analyze electricity consumption in different conditions. This paper combines the key technologies of electrical disciplines and information science. The model of consumers in smart grid is based on the agent-based modeling method and simulation system, to realize the consumer's respondence to the price of electric power. Plenty of price strategies are simulated in the model, according to the data which is obtained from the experiment. It provides the support of model and simulation system for generating the scientific price strategy in smart grid.
\end{abstract}

\section{Introduction}

As the development of global economic and the progress of science and technology, human society has gradually transform to the digital society, the continuous consumption of energy and environmental protection are becoming one of the common challenges that are humanity faces to[1]. As the advanced grid system structure, the smart grid becomes the development direction of electric power enterprise in the future [2].

Due to the particularity of electric power system, the traditional methods, such as physical modeling, will cost large. In the study of consumers' respondence and the process of electricity price system, scholars from all over the world prefer the classical methods of information science, to carry out the research work by data analyzing and modeling and simulation technology. Jia Dongli etc. [3] proceed related research of smart grid, by analyzing and contrasting the traditional power grid and smart grid of the United States, the European Union, China, made a in-depth discussion of the two-way interactive service concept and the theory of demand side management, further analyzed characteristics of consumers' respondence and the influence of electric price strategy. Sunil Luthra etc. [4] proceed the interaction of smart grid, in view of the development of smart grid, put forward the ideas of driving scheme, built the model based on the consumers to verify its feasibility. He Hongpeng etc. [5] proceed the modeling research and application of ontology technology in the field of power system, based on the resource description framework(RDF), through the in-depth analysis modeling instance smart grid related concepts. $\mathrm{O}$ ilkki etc. [6], proceed the simulation of different frequency control scheme of smart grid, came up with four different cases with details, assess the structure and applicability of the model.

Setting one fair price strategy has been a common concern to the government, the electric power enterprises and consumers, but due to the complexity of power system, the diversity of consumers' types, and the energy policy and environmental policy are always changing, all sorts of factors led to the complexity of the electricity business $[7,8]$. The structure of smart grid, technical complexity, performance in rich and diverse complexity embodied in physics and system complexity. Due to electric power has been the major energy pillar in the whole world, the development of the traditional power grid has reached a certain height, belongs to a very large scale complex network structure, smart grid, on the basis of traditional power grid, however, added digital information and network which can realize two-way flow, and the solar energy, wind power, nuclear power, hydropower and other renewable energy as the main power source[9], set established distribution 
contain the market of consumer, more complex than traditional power grid in physics.

The agent-based modeling method adopts the method of the underlying capture the upper system, by building the underlying model, perform the behavior of the whole system, makes the transition from micro to macro, conforms to the generate rule of the complex system. Through the observation and interaction among different agents, system can get all the agents' overall behaviors for data analyzing [10,11]. So agent-based modeling method is an effective method for the consumers' respondence to the price of electric price, putting forward the construction of model, and reinforcing simulation system development.

\section{The Overall Structure}

Based on the agent model and the research of consumer's behavior, the entire modeling process can be divided into the following four steps.

A. Understanding the global and local system. First consideration should be given by the prototype analysis and abstraction of character description, when creating the agent model shall consider about the scope of the local environment, that is not entirely visual environment. Agent-based modeling method requires clear rules of the system and the intelligent body, therefore accurate key parameters are important, and the contact between agents, to achieve the goal of maximum simulating grid consumer's behavior.

B. Methods. Based on the description of the system, on the basis of analysis of consumer's behavior study the power law, adopt the modeling method to construct module by modul. Based on agent technology, this paper emphasizes the change of the consumer's behavior, each agent will be given a series of rules and user agents will be divided into three categories: family, business and industry. According to user's characteristic and its respondence, key parameters and behavior rules were set up.

C. The design of interactive mechanism. By setting the parameter, agents have the realization of self-internal state transition, and the correlation between different agents, can be achieved through the interactive mechanism, the correlation between response and contains all the agent's overall trend.

D. Constructing the model of consumer as a whole. Understanding behavior of the whole system, determines the effective local behavior, after completion of analysis of prototype, constructing the local model conforms to the requirements of the system, establishing intelligent structure and adjusting for the details including the adjustment of main function and the parameter. Settings and state transition, can provide a model for the simulation experiment. Figure 1 is the processes of building the model.

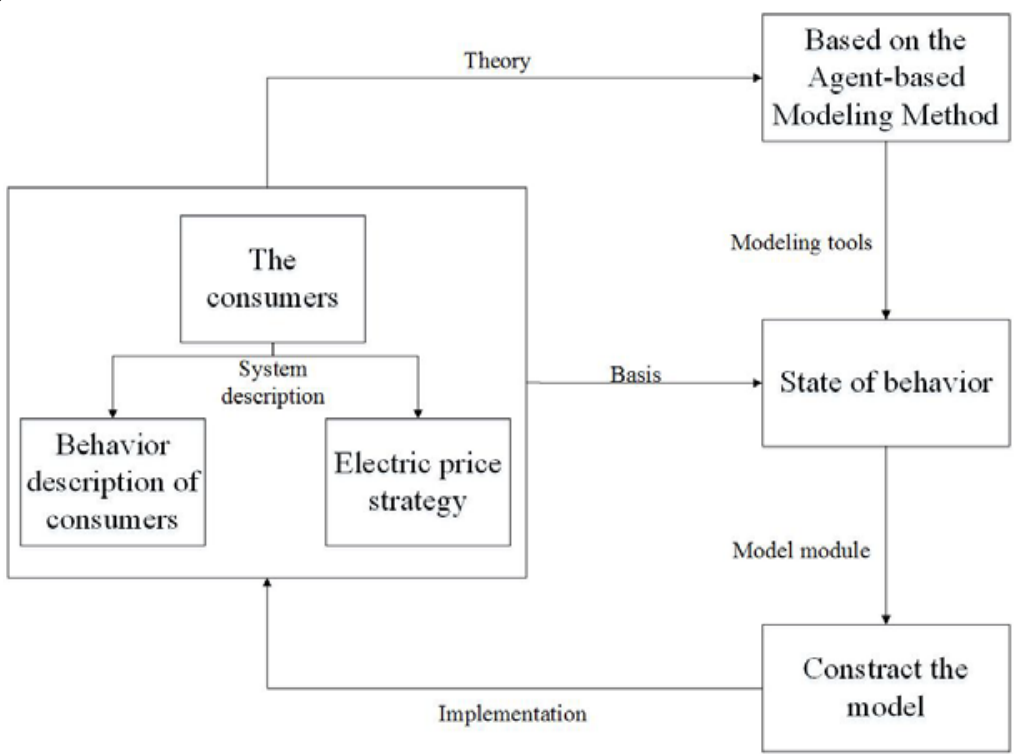

Fig.1. Processes of building the model

In order to facilitate consumer's behavior description, according to the characteristics, consumers 
can be divided into three types. Families mainly home users. Business mainly includes the medical and health, sports, education, scientific research, cultural entertainment, administrative office and commercial, service, finance, etc., due to its functional purpose, electricity utilization of time and load is stable. Electricity load base on the steady state. Industrial users is mainly manufacturing enterprises, textile, food, tobacco and other light industry and metallurgy, energy, building materials, chemical and other heavy industry, the demand is higher, to ensure the safety of the personnel and economic benefit of power supply, reliability requirements is extremely high, at the same time also has a high sensitivity for electricity price adjustment of relative, some enterprises could be changed by adjusting the shift of workers, work and rest time to avoid the peak season, choose low electricity during the production and reduce production cost effectively, expand the economic benefits of enterprises.

In addition to consumers, agent model would also include a part of the electric price strategy. Main influencing factors are electric price and power consumption, can make them respectively as control factors, in order to observe respond of the agent.

\section{Agent Model}

In this paper, this paper puts forward intelligent structure as shown in function (1).

$$
\text { Agent }=\{\text { Identity,Parameter,Connector,Status,Regulation,Environment }\}
$$

Among them, Identity is the unique identification. Parameter contains parameters and variables. Connector is intelligent body, used for receiving control information and output data. Status said all possible states. Regulation, by setting the rule of agent behavior, namely the state switch of incentives. Environment is the agent Environment.

This paper involving multiple intelligence structure, namely the multi-agent system (MAS), such as function (2) the said Agent groups.

$$
\text { MAS }=\{\text { Agents, Controller, Target }\}
$$

Among them, Agents is a collection of all agents in the system. Controller controls agent. Target said status to achieve of the change in the simulation.

In this paper, based on consumer's behavior model, agents can be divided into such as function (3) is shown in three categories.

$$
\text { Agents }=\{\text { Homes, Companies, Industries }\}
$$

where Homes, Companies, Industries, respectively have the different characteristic parameters.

Agent has two states: using and saving, respectively to simulate the user response in the process of fluctuation of electric price. Consumers in the implementation of acceptable price, namely normal state is used to simulate. When electric price beyond user acceptance, they will reduce total power consumption into saving state. These two kinds of situations can make a difference in power consumption, through statistical research trends in the number change.

As shown in figure 2 said agent of the two kinds of state transitions.

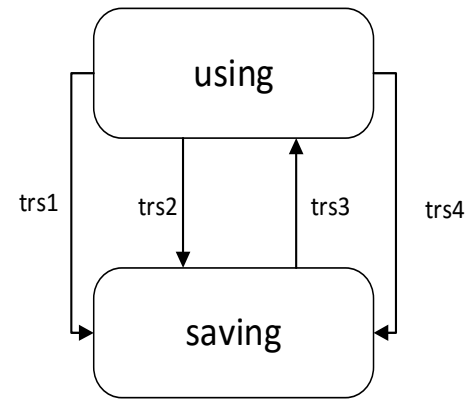

Fig.2. Behavior's process

Among them, trs1 said after the user power consumption exceeds a certain limit, happen response into a state of saving. Trs2 and tr3 respectively represent transformation rate between two states. Tr4 said another kind of switch mode of user state, namely when the price is more than the psychological price response occurs.

According to the above description of the user type, the user response price behavior, set up the 
key parameters reflecting agent features, for each type of user behavior is used to simulate again. Build the agent will be divided into three classes of Homes, Businesses, Industries, and set different parameters for it. Specific set quantity, dosage, changerate myprice. Number of which quantity is user group, user characteristics due to residents, should be significantly higher than the industry users and business users; Dosage reflect type daily power consumption of each user, to collect all kinds of power use of statistical data; Changerate reflect user response electricity price behavior is the main basis, according to each type of user electricity price sensitivity is different, its behavior in response time interval, the possibility is different; Myprice for the user's psychological price, that is, beyond psychological electricity users reduce electricity consumption.

Designed by the above behavior description, and data analysis, key parameters Settings as shown in table 1.

Table 1 Parameters

\begin{tabular}{llll}
\hline Type & Number & Rate & Price \\
\hline Homes & 200 & 0.1 & 100 \\
Businesses & 100 & 0.01 & 150 \\
Industries & 150 & 0.01 & 150 \\
\hline
\end{tabular}

In addition, the electricity price regulation system, set up the price of electricity parameter set in different Home_Price, Business_Price respectively, Industry_Price, residents said on the same day of the electricity utilization, commercial, industrial electricity price. In addition to the set Price parameters, the Max and min is another important parameter, Price said the Price, the Max and min are reflected power consumption quota, the user threshold value of the total power consumption.

\section{The Results of the Simulation}

This article USES the AnyLogic software modeling and simulation work, based on the completed user interface, control system interface, data analysis, hierarchical integration, operation simulation system for experiment data result diagram, as shown in figure 3.

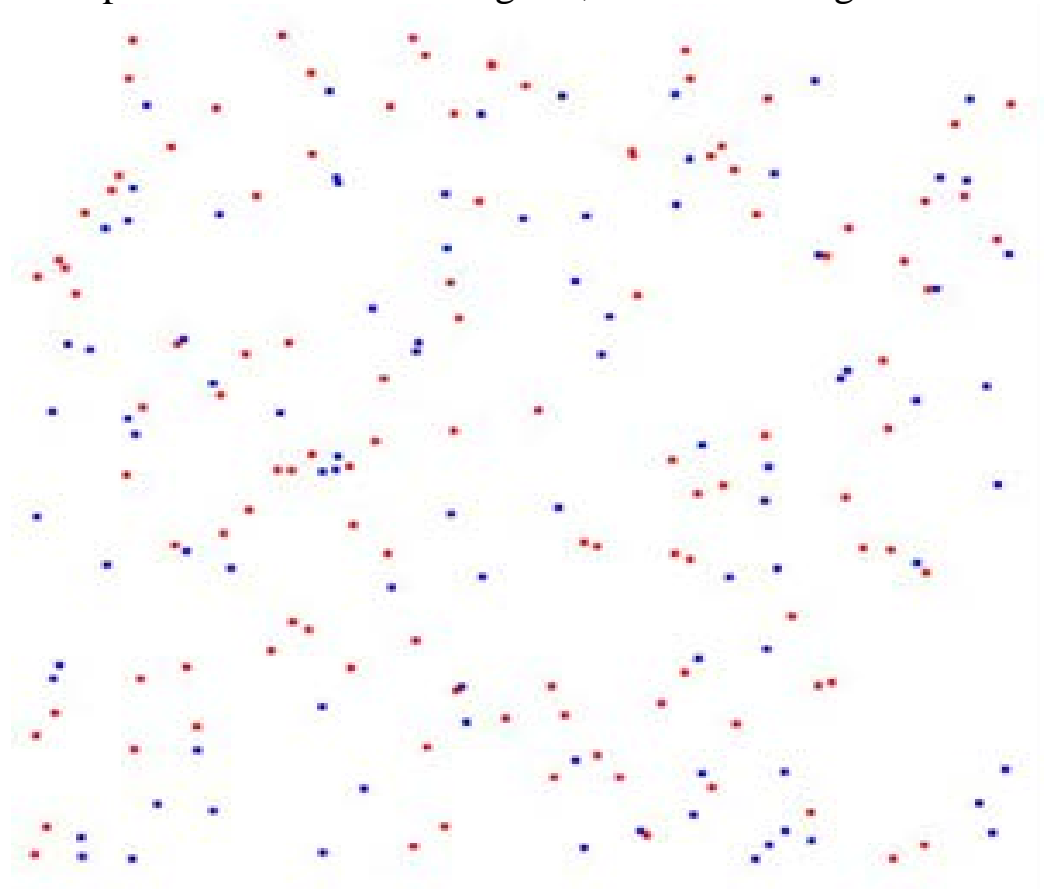

Fig.3. Planar figure of consumer

Two-dimensional graphic user status changes dynamic simulation, through the color to distinguish between different state transition of various types of users, can be seen as all residents and simulate a territory, as well as industrial users over a period of time response behavior.

During the running of model price changes as shown in figure 4. 

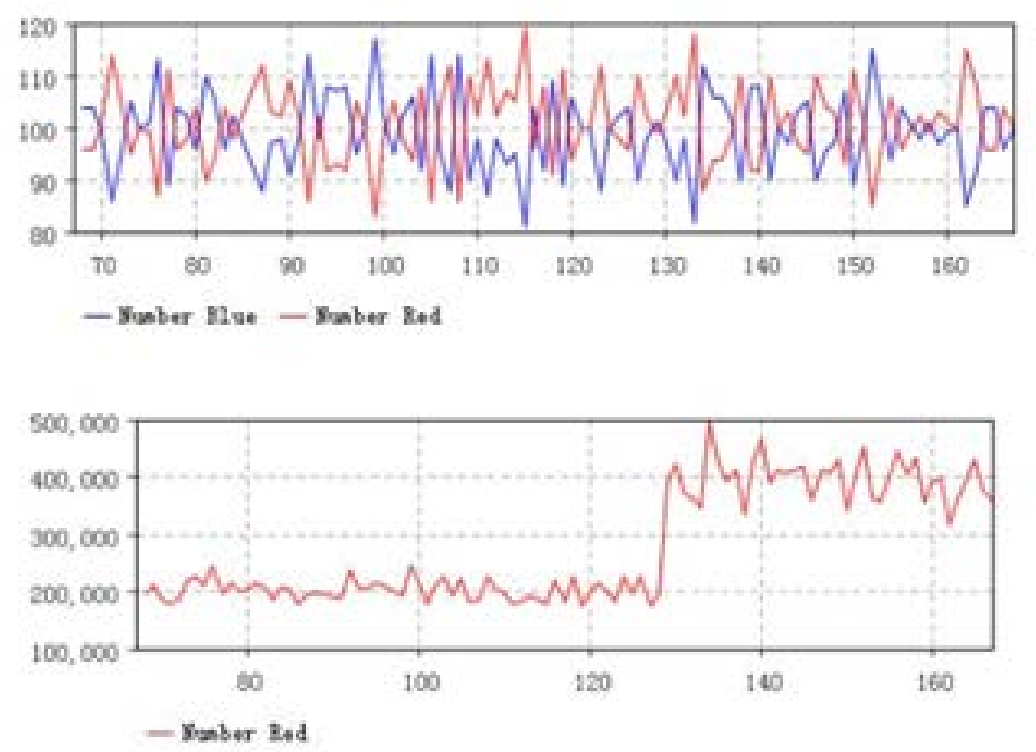

Fig.4. The results of simulation

The results of simulation data show the number of one kind of users during the model running, it can be observed under the influence of various factors, two states ongoing migration, such as increased by controlling the power threshold or electric price rise, that can be observed data decreased obviously, total electricity consumption and total electric price reduced at the same time.

\section{Conclusion}

Based on smart grid electricity price strategy optimization problem, considering the importance of grid user behavior research, agent based modeling and simulation technology, to construct a agent in the grid user model is put forward, through the development of simulation system is highly simulation grid users respond to price changes and power changes the behavior of statistics can be a period of time the user's state changes. This paper related research work and complete, not only research provides a theoretical method for power grid user response behavior, more to explore the optimal price strategy provided powerful support model and the simulation system, to promote the construction of smart grid and the full implementation of plays an important role and significance.

\section{Acknowledgement}

This work is supported by the National Natural Science Foundation of China (61374097 and 61473066), the Program for New Century Excellent Talents in University (NCET-12-0103), the Basic Science \& Research Fund of Northeastern University (N152303010) and of Northeastern University at Qinhuangdao (XNB201601), and the Science and Technology Research Project of Higher Education of Hebei Province (QN2016315).

\section{References}

[1] Jie Tang, Zhen Ren and Xiuzhen Hu. An Operable Peak-Valley Time-of-Use Tariff Setting Method for Demand Side Management [J]. Power System Technology, 2005, 29(22): 71-75.

[2] V.S.K.Murthy,Balijepalli, Vedanta Pradhan, S.A.Khaparde, R.M.Shereef. Review of Demand Response under Smart Grid Paradigm[C]//IEEE PES Innovative Smart Grid Technologies. India: IEEE, 2011:1-8.

[3] Marcel Arends, Paul H.J. Hendriks. Smart grids, smart network companies[J]. Utilities Policy, 2014, 28:1-11. 
[4] Joeri Naus, Gert Spaargaren, Bas J.M.van Vliet, Hilje M.van der Horst. Smart grids, information flows and emerging domestic energy practices[J].Energy Policy, 2014, 68:436-446.

[5] O.Kilkki, A.Kangasrääsiö, R.Nikkilä, A.Alahäivälä, I.Seilonen. Agent-based modeling and simulation of a smart grid: A case study of communication effects on frequency control[J]. Engineering Applications of Artificial Intelligence, 2014, 33: 91-98.

[6] Hania EI-Kanj Baitie, Tarek Selmi. Review of Smart Grid Systems Requirements[C]// Tenth International Conference on Ecological Vehicles and Renewable Energies. Australian: IEEE, 2015: 1-6.

[7] Deming $\mathrm{Xu}$, Jian $\mathrm{Xu}$ and Xiaoling Yao. Key Technology and Function Realization for Smart Grid [J]. Electrotechnics Electric, 011, 11: 35-41.

[8] M.Fadaeenejad, A.M.Saberian, Mohd.Fadaee, M.A.M.Radzi, H.Hizam. The present and future of smart power grid in developing countries[J]. Renewable.and Sustainable Energy Reviews, 2014, 29:828-834.

[9] Maria Carmen Falvo, Luigi Martirano. Technologies for Smart Grids : a brief review[C]// Electrical Engineering. Italy: IEEE, 2013:1-7.

[10] Shiban Kanti Bala, B.Chitti Babu. A Review on Development of Smart Grid Technology in India and its Future Perspectives[C]. India: IEEE, 2012: 1-6.

[11]Chaiyod Pirak, Tanayoot Sangsuwan. Recent Advances in Communication Technologies for Smart Grid Application: A Review[C]//Proceedings of the Electrical Engineering Congress. Thailand: IEEE, 2014:3-6. 